\title{
Trapping of strangelets in the geomagnetic field
}

\author{
L. Paulucci,, , J. E. Horvath, ${ }^{2}$ and G. A. Medina-Tanco ${ }^{3}$ \\ ${ }^{1}$ Instituto de Física - Universidade de São Paulo \\ Rua do Matão, Travessa R, 187, 05508-090, Cidade Universitária \\ São Paulo SP, Brazil \\ ${ }^{2}$ Instituto de Astronomia, Geofísica e Ciências Atmosféricas - Universidade de São Paulo \\ Rua do Matão, 1226, 05508-900, Cidade Universitária \\ São Paulo SP, Brazil \\ ${ }^{3}$ Instituto de Ciencias Nucleares, Universidad Nacional Autónoma de México \\ A.P. 70-543, C.U. México D.F., México
}

(Dated: October 27, 2018)

\begin{abstract}
Strangelets coming from the interstellar medium (ISM) are an interesting target to experiments searching for evidence of this hypothetic state of hadronic matter. We entertain the possibility of a trapped strangelet population, quite analogous to ordinary nuclei and electron belts. For a population of strangelets to be trapped by the geomagnetic field, these incoming particles would have to fulfill certain conditions, namely having magnetic rigidities above the geomagnetic cutoff and below a certain threshold for adiabatic motion to hold. We show in this work that, for fully ionized strangelets, there is a narrow window for stable trapping. An estimate of the stationary population is presented and the dominant loss mechanisms discussed. It is shown that the population would be substantially enhanced with respect to the ISM flux (up to two orders of magnitude) due to quasi-stable trapping.
\end{abstract}

\section{INTRODUCTION}

In a celebrated paper Witten [1] elaborated on the possibility [2, 3, 4] that systems composed of an deconfined Fermi gas of up, down and strange quarks could have a lower energy per baryon than iron, thus being absolutely stable. This hypothetical state (strange quark matter) could be created by weak interactions introducing the massive $s$ quark, if the energy "cost" of the mass is compensated by the availability of a new Fermi sea associated to this extra flavor, thus lowering the Fermi energy of the $u$ and $d$ quark seas.

Previous works have shown [5] that this stability may be realized for a wide range of parameters of strange quark matter (SQM) in bulk on the basis of the MIT bag model. Calculations also indicate that SQM can be absolutely stable within other models, e.g. shell model [6, 7], or not stable at all [8]. More recently, studies have indicated that a paired version of SQM, the CFL (color-flavor locked) state seems to be even more favorable energetically than the unpaired SQM, widening the stability window [9, 10, 11, 12].

For the description of finite size lumps of strange matter, (termed strangelets) a few terms have to be added to the bulk one in the free energy. A surface term suffices for $A \gg 10^{7}$, while other corrections are relevant for the lower masses (see [13] for a recent review). Large lumps will have essentially the same number of quarks of bulk matter, with a small depletion of the massive strange quark resulting in a net positive charge. This is a feature also expected for small chunks [6, 13], which thus

*paulucci@fma.if.usp.br resemble heavy nuclei.

In spite of theoretical controversies, it is generally agreed that the ultimate SQM proof must be provided by experiments. The experimental searches of strangelets started some 20 years ago and have been reviewed recently in [14, 15]. In addition to direct production of strangelets in heavy ion collisions [16, 17, 18, 19, $20,21,22,23,24]$, cosmic rays may contain primaries in this state of matter, which could eventually be detected directly or as a deposition in terrestrial matter 25, 26, 27, 28].

Several cosmic ray events have been tentatively identified in the past as primary strangelets (initially the Centauro events and the HECRO-81 experiment [29, 30, 31]) for they present features such as their high penetration in the atmosphere, low charge-to-mass ratio and exotic secondaries 32]. More recently, at least one event recorded from the AMS-01 experiment [33], a mass spectrometer aboard the shuttle Discovery during a 10-day flight in 1998, is being considered as possible detection of a strangelets. While it is tempting to identify the primary as a strangelet, the inevitable shell effects complicate the analysis and preclude any firm conclusion as yet 34]. It is not clear until today to what extent the anomalous events can be originated by ordinary primaries or rather forcefully require a truly exotic origin.

Considering the question of existence of strangelets among cosmic ray primaries, a few injection (production) scenarios have been considered. Witten originally suggested the merging of compact stars as a likely site [1]. In principle, injection spectra and the total mass in the galaxy may be calculated knowing the rate of the events and the total ejected mass in each of them. These estimates are subject to some caveats, for example, while the number of merging systems has been revised upwards 
[35], numerical work has shown that a substantial ejection of matter is not guaranteed [36] in a strange starblack hole system, and the situation is unclear in the case of a fully relativistic SS-SS system, which has only been partially addressed [37] because the calculations had other goals. On the other hand, strange matter formation in type II supernova [38] has been preliminarily explored and in these events a small fraction of strange matter may be ejected. A numerical analysis has shown that the possible quark matter component of cosmic rays primaries is compatible [39] with models in which strangelets are ejected in either scenario.

While an uncertain flux from this "contamination" of the ISM is expected [40], we would like to discuss in this paper another likely site to search for strangelets of cosmic origin. Much in the same way heavy nuclei are present in the earth's magnetosphere bouncing between magnetic mirror points, strangelets could also become trapped in specific regions of the magnetosphere and their number density increased respect to the ISM flux, provided some conditions for their capture by planetary magnetic fields are met. This phenomenon is analogous to the Van Allen belts, and has been first suggested in a former study [41]. A handful of experiments have probed the magnetosphere by measuring the fluxes of the so-called "anomalous" cosmic ray nuclei, and may already place interesting limits to strangelets as well. Overall the existence and nature of exotic primaries is an important issue. In addition to former and ongoing searches, there will be a mass spectrometer placed at the International Space Station, the AMS-02 experiment [42, 43], with one of its goals to help the identification of this exotic component, of crucial importance in testing the validity of the Bodmer-Witten-Terazawa conjecture. We substantiate below the strangelet belt idea, discuss the main features of this population, and advocate for a search of this exotic component at definite sites within existing uncertainties based on these calculations.

\section{STATES OF IONIZATION AND ELECTRONIC RECOMBINATION OF STRANGELETS IN THE ISM}

As is well-known, unpaired (also referred as "normal" in this work) SQM in bulk contains light $u, d$ and massive $s$ quarks in $\beta$-equilibrium. Because of the depletion of the more massive $s$ quark, a small fraction of electrons is also present to maintain charge neutrality. On the other hand, SQM in a paired CFL state is automatically neutral, since the equal number of flavors is enforced by symmetry [44. Actually, a small positive charge is present because of the smaller abundance of $s$ quarks near the surface in CFL strangelets [13]. Therefore it is natural that CFL strangelets will be surrounded by an electronic cloud in order to neutralize its total charge, forming an exotic atom. The same happens for normal strange matter if the strangelet radius is smaller than the electron Compton wavelength, a condition satisfied whenever $A \ll 10^{7}$.

In the following and throughout the whole analysis presented here, the strangelet rest mass will be assumed to be $\epsilon_{0} A \sim(930 \times A) \mathrm{MeV}$, with $\epsilon_{0}$ the asymptotic value of the energy per baryon of strange quark matter. We will not consider the fact that the energy per baryon number decreases with $A$ in sophisticated model calculations, given that the uncertainties found in other parameter choices are expected to be much larger than the error associated with this approximation. Also the strange quark mass is considered to be $m_{s}=150 \mathrm{MeV}$ and the coupling gap of CFL strange quark matter, $\Delta=100 \mathrm{MeV}$ in this exploratory study. With these assumptions, the net positive charge of strangelets is given approximately by $Z=0.1 A$ (low baryon number regime) in the MIT bag model approach for normal strange matter and $Z=0.3 A^{2 / 3}$ for the CFL model.

Strangelets from whatever astrophysical injection event would travel through the interstellar medium and become ionized by collisions. A simple analysis to evaluate the degree of ionization of semi-relativistic strangelets surrounded by electronic clouds due to these interactions was performed in a Bohr atom approximation. Strangelets are partly neutralized by electrons from the excitation of the vacuum if $Z \gg 100$ [45], but for all cases of interest in this work the baryon number range is such that we do not have to deal with this effect.

We considered a two-body collision (incident electron - electron in the strangelet cloud) instead of a multibody problem, which would be much more difficult to handle. The stripping interactions are mainly due to electrons with a Maxwellian speed distribution at a temperature of $\sim 100 \mathrm{~K}$, an average condition of electrons in the ISM.

The results are shown in figure 1 for strangelets with total energy of $1 \mathrm{GeV} / \mathrm{A}$. Considering the average density in the interstellar medium to be 1 particle $/ \mathrm{cm}^{3}$, the mean free path for an electronic collision which may or may not result in ionization is of the order of $10^{15} \mathrm{~cm}$, which is very short on astronomical standards.

The ionization degree became stable within a travelled distance of a few $p c$ for $1 \mathrm{GeV} / \mathrm{A}$ strangelets. For ultrarelativistic strangelets (i.e., of the type of candidates that would produce a Centauro event [46] $E / A \sim T e V)$ the calculations indicate always full ionization. Furthermore, according to the model proposed by Werner and Salpeter 47] for the radiation flux in the ISM, the influence of the radiation field on ionization of strangelets will be negligible unless the strangelet trajectory crosses a region containing very energetic photons (i.e. the surroundings of a Wolf-Rayet, $\mathrm{O}$ and $\mathrm{B}$ stars and/or regions of stellar formation).

We acknowledge that a Bohr atom treatment is a crude approach for the electron distribution around the strangelet, since it does not include quantum corrections as important as the spin-orbit coupling and non-local effects, nor relativistic corrections for many electrons bodies $(Z \geq 40)$. There is no general expression for these corrections applicable in the case of atoms with many 

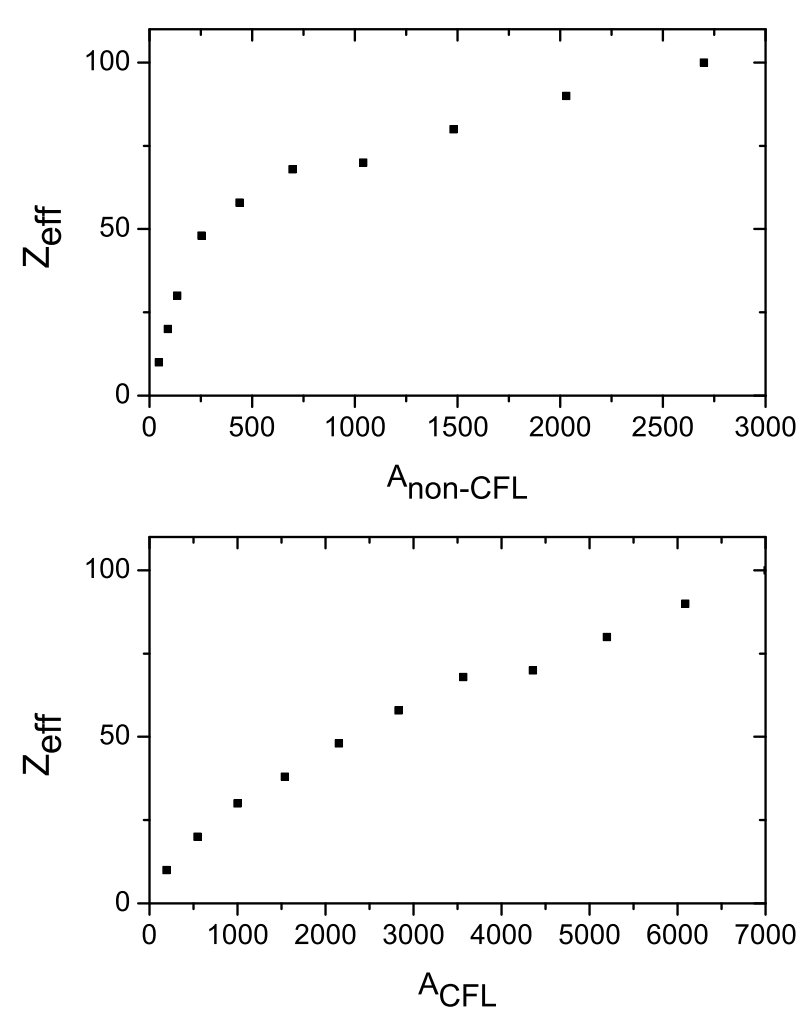

FIG. 1: Strangelet effective charge $(1 \mathrm{GeV} / A)$ versus the baryon number $A$ for normal (upper panel) and CFL (lower panel) strange matter after interaction with electrons in the interstellar medium.

electrons though; the existent models (e.g., Hartree-Fock calculations) are restricted to atoms with few electrons, the same happening for experimental corrections. In this way, the calculations presented here are rough estimates, showing the general trend of the effects rather than providing precise numerical values.

For low-energy particles the electronic capture can be as important as the ionization process thus far discussed. An approximate cross section for the capture of electrons of velocity $v$ by a charged particle of atomic number $Z$ is given as [48] [49]

$$
\sigma_{c}=Z^{2} 2^{2 / 3} \alpha^{4} \frac{h^{2} \nu^{2}}{m_{e}^{2} v^{2} c^{2}}\left(\frac{m_{e} c^{2}}{h \nu}\right)^{7 / 2} \times 6.65 \times 10^{-25} \mathrm{~cm}^{2}
$$

where $h \nu \approx E_{e}$ for $E_{e}>>I, I$ and $E_{e}$ being the electron energies while bound to the nucleus and free in the ISM, respectively, and $m_{e} c^{2}$ is the electron rest mass. This form of the cross-section for radiative recombination is obtained relating the capture of a bare nucleus of charge $Z e$ with the capture into the corresponding state of a hydrogen atom, which is proportional to the energy of the gamma emitted in the process and also to the crosssection for the absorption of a quantum of frequency $\nu$ by a $H^{-}$ion resulting in emission of an electron of velocity $v$, and inversely proportional to the momentum of the electron absorbed. In the case of a partially screened nucleus, the cross-section is still given approximately by equation (11), though a special calculation must be performed to obtain the cross-section for capture into an orbital with quantum number $n_{0}$, usually given in tables for ordinary nuclei.

The "atom" or "ion" formed by capturing an electron may also lose this electron in further interactions. For light materials the cross section for electron loss can be approximately expressed for $v>v_{0}[50]$ as

$$
\sigma_{l}=8 \pi a_{0}^{2} Z^{-2}\left(\frac{v_{0}}{v}\right)^{2}
$$

where $a_{0}=\hbar^{2} / m e^{2}=0.53 \times 10^{-8} \mathrm{~cm}$ is the Bohr radius and $v_{0}=e^{2} / \hbar$, whereas for intermediate $Z$ materials

$$
\sigma_{l}=\pi a_{0}^{2} Z^{-1}\left(\frac{v_{0}}{v}\right)
$$

because of the screening effect.

In summary, a comparison of eqs.(1), (2) and (3) shows that electronic capture would only be important for high $\mathrm{Z}$ strangelets, precisely where this simple picture can no longer be applied due to vacuum excitation effects. That corresponds to a region in baryon number which we believe to be of minimum relevance to the trapped population.

In summary, these results indicate that we can assume total ionization as a good approximation to incoming ISM strangelets that could form an ionization belt in the magnetosphere.

\section{CAPTURE OF STRANGELETS IN THE GEOMAGNETIC FIELD}

The motion of ionized strangelets in the earth magnetosphere can be studied by applying the Störmer theory in a dipolar magnetic field. The movement analysis can be made in terms of the geomagnetic latitude and the $L$ parameter, where $L$ is the equatorial distance of a field line to the axis of the dipole measured in units of the earth radius.

The geomagnetic field is not a pure dipole field. Instead, most magnetic models used for studying it include nearly 50 terms for describing the potential field from which the magnetic field is obtained in a sum of Legendre functions multiplied by oscillatory coefficients in the azimuthal variable. Since the potential field has a $r^{-(n+1)}$ dependence, the importance of high-order terms decreases rapidly as one moves away from the earth surface. In this way, the $n=1$ term, i. e., the dipole term, is 
the lowest but dominant term, and most features of the trapped radiation theory are analyzed based on a dipole field.

Charged particles with energy of order of $\mathrm{MeV}$ in the inner part of the magnetosphere $(L \ll 10)$ rotate with a much higher frequency than that of typical geomagnetic field variation (which varies in time scales of, at most, few minutes). Under these conditions, the magnetic moment is a conserved quantity (adiabatic invariant). Therefore particles with high enough magnetic moments become trapped in the dipolar field lines of the geomagnetic field, with mirror points placed near the earth poles.

Particles with mirror points that allow penetration in the earth atmosphere can be lost via collisions with atoms. All the particles with mirror points placed inside the earth radius are obviously lost, meaning that particles with $\left|\alpha_{e q}\right|<\alpha_{E}$ or $\left|\pi-\alpha_{e q}\right|<\alpha_{E}$, where $\alpha_{e q}$ is the equatorial pitch angle, are inside the earth loss cone.

We will consider collisions mainly with the neutral nitrogen molecule $\left(N_{2}\right)$. The probability of interaction of trapped particles which penetrate the atmosphere (suffering collisions losses) can be taken as

$$
P(s)=1-e^{-s / \lambda(s)}
$$

at a certain point $s$, since each process is probabilistically independent, being $\lambda$ the particle mean free path. Generalizing the previous equation, it is necessary to integrate over the particle path. Assuming that all the strangelets which collide with particles in the atmosphere are eventually removed from the trapped flux, we express the escape probability as

$$
P_{e s c}=1-e^{-\int_{s} \sigma\left[n\left(s^{\prime}\right)+s^{\prime} \frac{d n}{d s^{\prime}}\right] d s^{\prime}}
$$

where $d s=L R_{E} \cos \lambda \sqrt{1+3 \sin ^{2} \lambda} d \lambda$ is the arc along a field line, $\sigma$ is the particle cross-section and $n(s)$ is the density of particles in the atmosphere at a certain point $s$ of the strangelet's path. Since strangelets are hadrons we may take their relevant interaction cross-section to be geometrical $\left(\sigma \propto A^{2 / 3}\right)$.

The calculated loss cone for strangelets, assuming an exponential profile of the atmospheric density is shown in figure 2 for different $L$ reflecting collisions with atmospheric particles and the non-existence of a suitable mirror point. It indicates, as expected, that the smaller the equatorial pitch angle the easiest it is to remove a trapped particle.

In order for a particle to penetrate a certain region in the magnetosphere its energy must be enough to overcome the local geomagnetic cutoff rigidity. A solution was found by Störmer [51] describing a special case of what he called the "forbidden cone", which gives the geomagnetic cutoff rigidity. In this way, the condition a

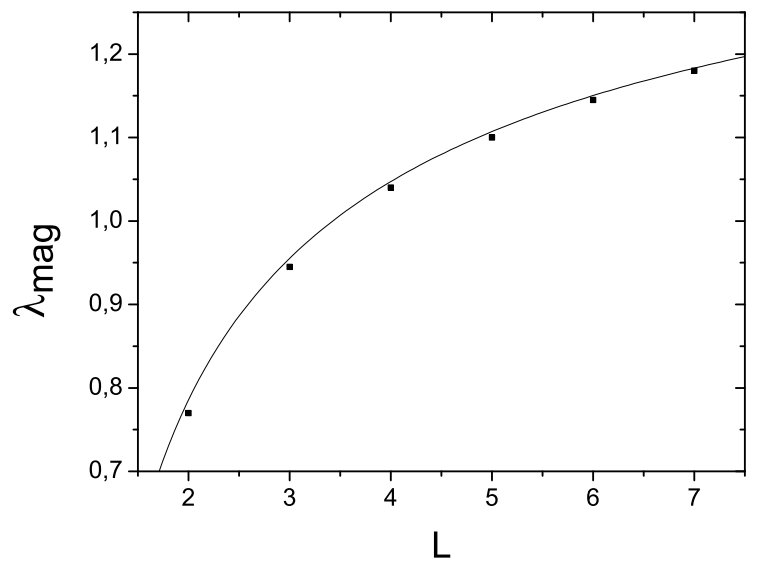

FIG. 2: Loss cone for strangelets in the geomagnetic field, i. e., particles with mirror point placed at geomagnetic latitudes above the square dots (coming from the analysis of collisions with atmospheric particles) are eventually removed from the trapped population. The full line represent mirror points at the earth surface in the dipole field approximation.

particle must fulfill to have access to a given region of the magnetosphere can be written as 52 ]

$$
R_{\text {particle }}>\frac{59.6 \cos ^{4} \lambda}{L^{2}\left[1+\left(1-\cos \gamma \cos ^{3} \lambda\right)^{1 / 2}\right]^{2}} G V
$$

where $\lambda$ is the latitude and $\gamma$ the arrival direction of the particle (east - west).

In the analysis of charged particles trapped in a magnetic field it is usually considered that the motion of a given particle is a composition of three different motions: the bouncing motion of a guiding center along the magnetic field line; the rotational motion of the particle itself around that guiding center; and the longitudinal drift of the guiding center. In this way, the condition for a triply - adiabatic motion is that the magnetic field intensity must vary very slowly around a cyclotron orbit, imposing a maximum energy allowed for stable trapping. The condition that must be imposed for the cyclotron radius at the equator is given by

$$
\left.R_{C}\right|_{\text {equator }}=\left.\frac{p_{\perp}}{q B} \ll \frac{B}{\left|\nabla_{\perp} B\right|}\right|_{\text {equator }}
$$

Figures 3 and 4 show those bounds for normal and CFL strangelets, respectively, for $L=2$ in addition to the minimum baryon number which is required for strangelet stability [7]. The existence of a minimum baryon number is expected in all models of SQM because the energy needed for producing the system increases as the baryon number decreases, till it reaches a value above which the 
strange matter is unstable. The value adopted has been $A_{\min }=30$ (shown with a vertical line) and may be trivially altered for any other figure. Strangelets with very high baryon number, though allowed for stable trapping, are not likely to be statistically significant for detection in the magnetosphere due to a substantial decrease of the interstellar flux expected as the baryon number increases.

The upper bound (7) has been enforced in our calculations to a $10 \%$ confidence level according to observations of anomalous cosmic rays $L$-shell distributions [53], and we considered $E_{\perp} \sim E$, which means we are actually underestimating the number of particles that could be stably trapped in the geomagnetic field. Obviously, the geomagnetic cutoff curve must be below the adiabaticity criteria for stable trapping to occur. This is not the case for small latitudes, but there is a narrow "window" in latitude starting slightly above 30 degrees at $L=2$ for strangelets from the ISM flux to fulfill the conditions of capture and accumulate in regions labelled by the $L$ parameter. However, the number of accumulated particles is still interesting, as shown in the next section.

When this calculation is repeated for the case of CFL strangelets, the region allowed for stable trapping for CFL strangelets has a different shape than that for normal strangelets. This feature is due to the strong dependence of the charge upon $A$ of normal strangelets $(Z \propto A)$ resulting in constant values if one considers momentum per baryon number, whereas for the CFL strangelets charge is almost independent of $A$, leading to $\mathrm{a} \sim A^{-0.9}$ dependence in the momentum per baryon number variable.

\section{A. Trapped strangelet population}

Even though strangelets can be captured and trapped in the earth's magnetic field, we must evaluate the possible maintenance of a strangelet population to check whether there is an increase of the flux. For this purpose, we must consider losses mechanisms.

In addition to the already analyzed losses by collisions with neutral atmospheric particles, we have considered the inward drift driven by asymmetric fluctuations of the geomagnetic field as a dominant mechanism to diminish the strangelet population.

We will not consider in this work direct pitch angle diffusion. Because of their large mass, strangelets are less likely to be scattered appreciably in pitch angle by collisions. The net result of multiple collisions with atmospheric particles would be a reduction in the strangelets kinetic energy to thermal values and minor changes in their pitch angle. Since we have already considered that particles bouncing at a radial distance from the surface of the earth below the atmosphere height scale (derived in section II) would be eventually removed, we are in fact replacing a diffusion equation in the $\cos \left(\alpha_{e q}\right)$ variable for a constant loss term (a sink function) directly related, though not formally assigned, to pitch angle diffusion.
Radial diffusion must proceed by fluctuations in the third invariant $\phi$, which is proportional to $L^{-1}$, due to changes in the electric or magnetic fields that are more rapid than the particle drift frequency. Because the gyration and bounce periods are much shorter than the drift period, the first and second adiabatic invariants are less likely to be affected by many of these field perturbations.

Guided by the existing calculations and observations for anomalous cosmic ray nuclei (hereafter ACR) trapping, we have considered third invariant diffusion due asymmetric fluctuations in the geomagnetic field, which is mainly driven by the solar wind pressure (sudden compression and slow relaxation of the geomagnetic field).

The diffusion coefficient $D_{L L}$ is determined theoretically by taking two consecutive steps [54]. First, one has to evaluate the radial displacement suffered by a particle under the influence of the field disturbance, which is an idealized model of the real disturbances occuring in the geomagnetic field. The following procedure is taken in order to obtain the diffusion coefficient as a function of the statistical features of the disturbances alone. It consists of squaring this displacement and taking the average over several disturbances randomly occurring in time and over all possible particle's initial longitudes.

The diffusion coefficient due to magnetic field fluctuations for equatorially trapped particles, with the assumption of efficient phase mixing [54] can be expressed as

$$
D_{L L}^{M}=\frac{\pi^{2}}{2}\left(\frac{5}{7}\right)^{2} \frac{R_{E}^{2} L^{10}}{B_{0}^{2}} \nu_{d r i f t}^{2} P_{A}\left(\nu_{d r i f t}\right)
$$

where $P_{A}(\nu)$ is the power spectral density of the field variation evaluated at the drift frequency. For offequatorial particles, the diffusion coefficient presents an exponential decay with latitude.

Already in the case of nuclei, it is known that the complex geometry and inhomogeneities in the geomagnetic field make quantitative calculations ambiguous. The observed values of the diffusion coefficient and their $L$ dependence will change with global magnetic activity, and magnetic disturbances are known to vary appreciably with time. We have assumed a $\nu^{-2}$ dependence of the power spectral density for simplicity [54]. The loss of more detailed information associated with this approximation is that the diffusion coefficient becomes independent of the energy of the particle entering the geomagnetic field. In this case, the diffusion coefficient have a strong dependence on the McIlwain parameter $\left(D_{L L} \propto L^{10}\right)$ 60]. This indicates that its influence is very important for particles trapped at higher $L$-shells.

Typical values for changes in the trapped population distribution ranges from a few hours at $L=6$ to hundreds of days at $L=2$. Therefore if strangelets are captured by the geomagnetic field their density must be higher for lower values of the $L$ parameter, which may result in a substantial increase of this population compared to the ISM flux. 

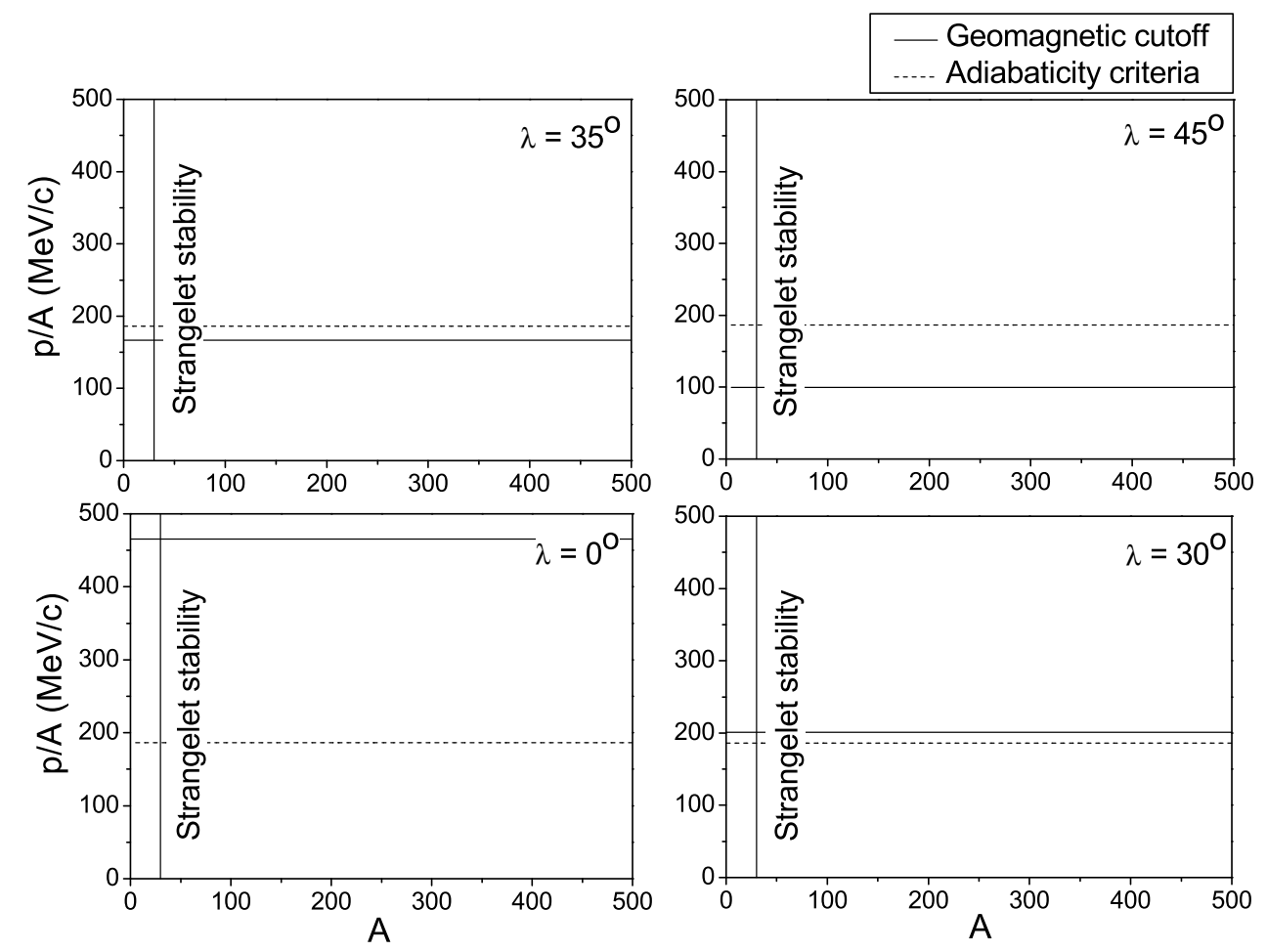

FIG. 3: Restriction curves (6) and (7) for $L=2$ in the baryon number vs. momentum plane for normal strangelets incident from the east $(\gamma=\pi)$ for different incident directions (reminding that field lines at $L=2$ penetrate the earth surface at $\lambda=45^{\circ}$ in the dipole model).

Some other losses mechanisms are of less importance in short time scales, but have influence on long time scales, thus lowering the residence time for trapped particles. This includes electrical drift-resonant interactions between particles and fields, especially in the pulsation frequency or VLF range [55]. Those phenomena are highly affected by the solar wind activity.

The diffusion equation has been employed to study the trapped strangelet flux

$$
\frac{\partial f(\mu, J, L)}{\partial t}=\frac{\partial}{\partial L}\left[\frac{D_{L L}}{L^{2}} \frac{\partial}{\partial L}\left(L^{2} f(\mu, J, L)\right)\right]
$$

where $f$ is the distribution function, $D_{L L}$ is given by equation (8) and $\mu$ and $J$ are the adiabatic invariants magnetic moment and integral invariant, respectively. The relation between the distribution function and the flux may be given by $j(E, \alpha)=p^{2} L^{2} f(\mu, J, L)$. A stationary population requires $\partial f / \partial t=0$, i. e., the assump- tion that the source and loss terms are instantaneously balanced is valid.

We assume a steady strangelet injection from the interstellar medium at $L=6$ (the position of the maximum distribution function is very insensitive to the chosen Lshell parameter for this boundary condition) and derive the distribution function shape between this maximum and $L \approx 1.05$ where it is null (atmosphere particle interaction height), shown in Figure[5, We are not considering diffusion in pitch angle due to interaction of particles with electromagnetic waves caused by field variations, which alters the first adiabatic invariant.

The calculations were carried on with two values of the flux from the ISM reaching the outer magnetosphere.

The first one, which will be called "standard", is the one that assumes the standard cosmic ray dependence on the strangelet flux, $E^{-2.5}$. The total ISM strangelet flux that reaches the earth as estimated by Madsen [40] for a binary strange star system coalescence scenario is given by

$$
F \approx 2 \times 10^{5} m^{-2} y r^{-1} s r^{-1} A^{-0.467} Z^{-1.2} \max \left[R_{S M}, R_{G C}\right]^{-1,2} \Lambda
$$




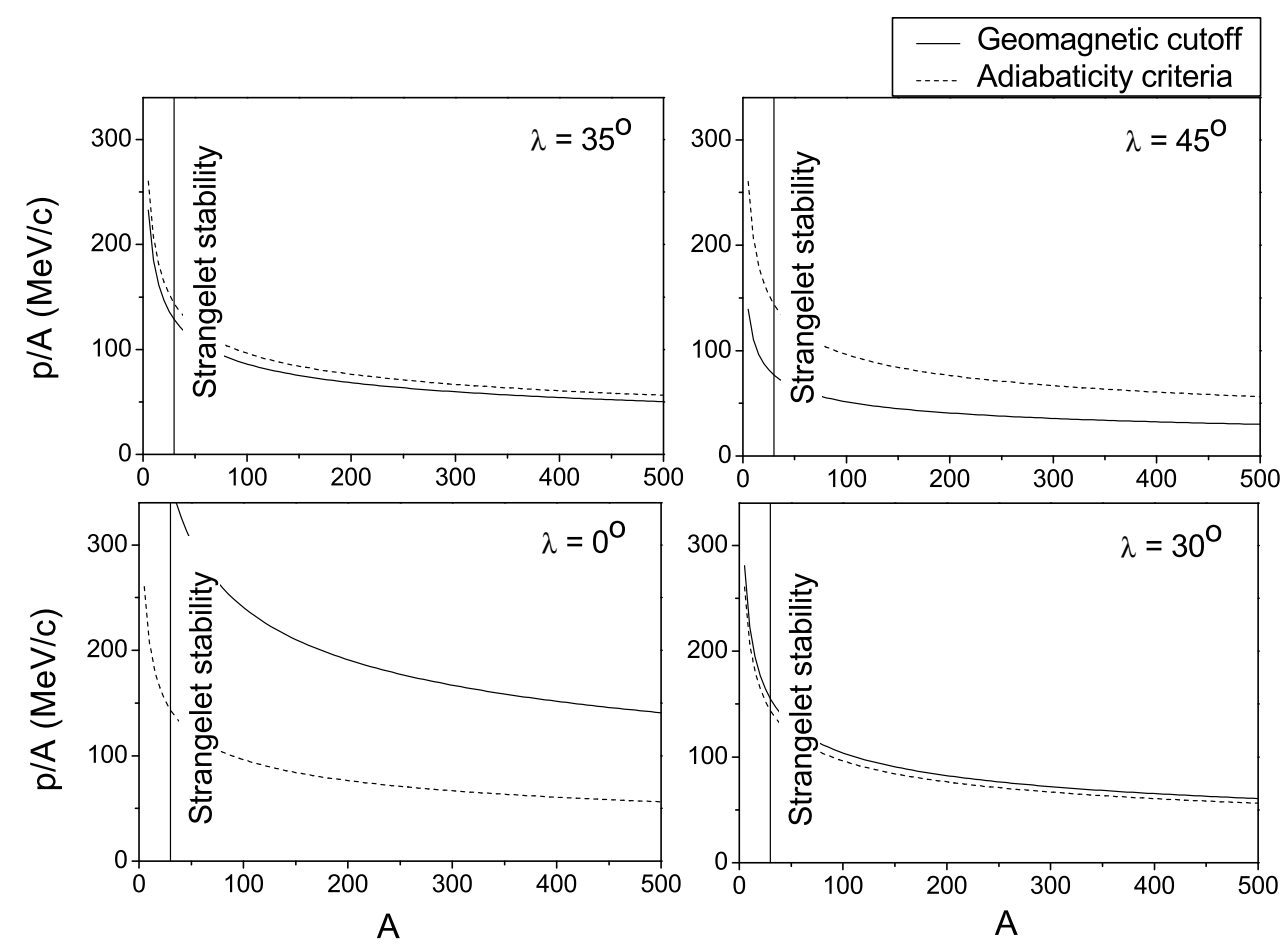

FIG. 4: The same as Figure 3 for CFL strangelets.

where $R_{S M}$ and $R_{G C}$ are the solar modulation and geomagnetic cutoff rigidities, respectively, and $\Lambda$ is an uncertain parameter assumed to be of $O(1)$. In this way, the whole flux is fitted with a $E^{-2.5}$ dependence with the constraints of minimum and maximum energy respecting the values $R_{\min }=5 M V A / Z$ and $R_{\max }=10^{6} G V$ [40].

The second calculation, which will be called "improved", considers a more detailed characterization of the differential flux, where for the region of interest in this work (rigidities of few GV), the strangelet flux actually increases with a slope of $R^{1.8}$. This flux was obtained from a fit to reference [40].

In either way, the flux entering the region of the magnetosphere at $L_{\max }$ has to fulfill the restrictions imposed for stable trapping in the pitch angle and geomagnetic latitude of incidence

$$
F_{\text {in }}=\int_{\lambda_{\min }}^{\lambda_{\max }} d \lambda P(\lambda) \int_{\alpha_{\text {loss cone }}}^{\pi / 2} d \alpha_{e q} P\left(\alpha_{e q}\right) \times F
$$

The efficiency factors, $P(\lambda)$ and $P\left(\alpha_{e q}\right)$ may be easily identified: $P(\lambda)$ gives the fractional area of the spherical section suitable for trapping discussed previously

$$
P(\lambda)=\frac{2 L^{2}(-\cos \lambda) \int_{0}^{2 \pi} d \phi}{2 L^{2} \int_{0}^{\pi / 2} \cos \theta \int_{0}^{2 \pi} d \phi}
$$

where the factor 2 comes from the symmetry in $\theta$ for both hemispheres (north, south). $P\left(\alpha_{e q}\right)$ limits the number of particles entering the specific region of the magnetosphere with an appropriate pitch angle to avoid the loss cone as already discussed. We have also assumed an isotropic flux of particles, since there is no theoretical prediction pointing to any anisotropy in the arrival direction of strangelets, which means that $j_{0}\left(\cos \alpha_{e q}\right)=$ constant is a reasonable hypothesis:

$$
P\left(\alpha_{e q}\right)=4 \frac{\alpha_{e q}}{\int_{0}^{\pi / 2} \alpha_{e q} d \alpha_{e q}}
$$

where the factor 4 stands for the symmetry in the condition for a given particle to belong to the loss cone: $\left|\alpha_{\text {eq }}\right|<\alpha_{\text {loss cone }}$ and $\left|\pi-\alpha_{\text {eq }}\right|<\alpha_{\text {loss cone }}$.

Solving the differential equation (9) and obtaining the corresponding flux inward (in the - $\hat{\mathrm{e}}_{r}$ direction) for every $L$, it is possible to determine the mean particle density at a given shell and, in this way, the trapped strangelet flux. 


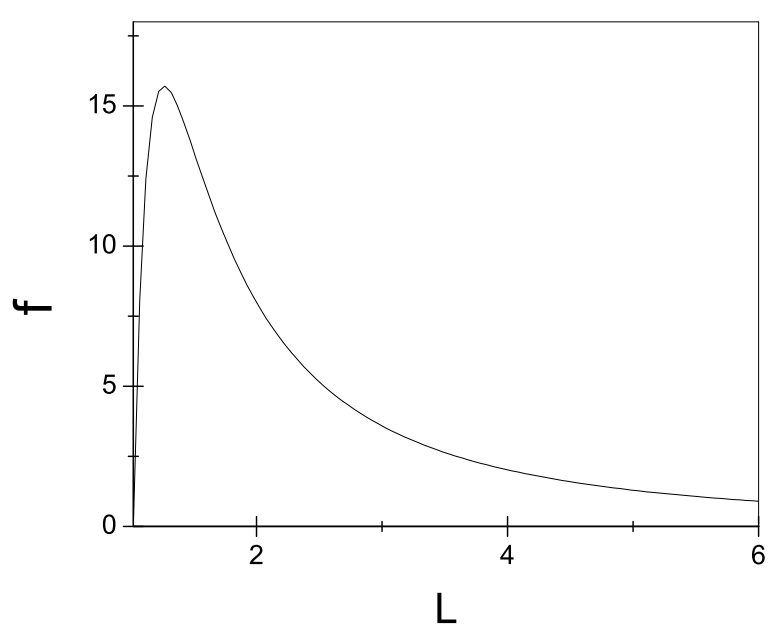

FIG. 5: The distribution function (in arbitrary units) for strangelets trapped in the geomagnetic field as a function of $L$ is obtained as the solution for the differential equation 9 with the boundary conditions $f\left(L_{\max }\right)$ given by the incoming flux (see text for details) and $f\left(L_{\text {min }}\right)=0$ (corresponding to the height scale of interaction with atmospheric particles). The position of the peak (around $L=1.3$ ) does not change with the change in the $A / Z$ relation (CFL and nonCFL strangelets) nor with a change in the energy and A of the strangelets.

\begin{tabular}{|l|l|l|}
\hline & $L=1.3$ & $L=2$ \\
\hline Normal & $1.28 \times 10^{-15}$ & $4.34 \times 10^{-17}$ \\
\hline CFL & $3.95 \times 10^{-14}$ & $1.34 \times 10^{-15}$ \\
\hline
\end{tabular}

TABLE I: Mean particle flux in units of part $\mathrm{cm}^{-2} \mathrm{~s}^{-1} \mathrm{sr}^{-1}(\mathrm{MeV} / \mathrm{A})^{-1}$ for strangelet stationary population at $L=1.3$ and $L=2$ calculated with the "standard" flux.

The results are summarized in Tables 1 and 2 for $L=2$ (ACR belt location) and $L=1.3$ (location of the maximum of the distribution function) for the example of strangelets of $A=100$ and energy corresponding to $R=1 G V$.

The position of the peak of the distribution function in the geomagnetic field (around $L=1.3$ ) is quite robust, it

\begin{tabular}{|l|l|l|}
\hline & $L=1.3$ & $L=2$ \\
\hline Normal & $3.83 \times 10^{-14}$ & $1.3 \times 10^{-15}$ \\
\hline CFL & $1.64 \times 10^{-13}$ & $5.55 \times 10^{-15}$ \\
\hline
\end{tabular}

TABLE II: Mean particle flux in units of part $\mathrm{cm}^{-2} \mathrm{~s}^{-1} \mathrm{sr}^{-1}(\mathrm{MeV} / \mathrm{A})^{-1}$ for strangelet stationary population at $L=1.3$ and $L=2$ calculated with the "improved" flux. does not appreciably change with the change in the $A / Z$ relation (CFL and normal strangelets), nor with a change in the energy and baryon number of the strangelets. This could be a consequence of the assumption of the power spectral density as being proportional to $\nu^{-2}$, what renders the diffusion coefficient independent of the particle energy, therefore modifying the energy of the particles does not affect their diffusion properties.

We observe that the trapped population is slightly more favored if strange quark matter is in the CFL state, the difference between the trapped fluxes for the two species increases with decreasing energy exponent in the incident flux. It happens due to the dependence on the baryonic number of the interstellar flux of strangelets (eq. 10). Since the rigidity interval for stable trapping is the same for both states for it only depends on the geometrical characteristics of the geomagnetic field, the difference on the number of particles trapped strongly depends on the difference in the incoming flux. This dependence of the integrated flux on the number of baryons that can be expressed as $F_{I S M} \propto(0.125)^{-1.2} A^{-1.667}$ and $F_{I S M} \propto$ $(0.3)^{-1.2} A^{-1.267}$ for normal and CFL strangelets, respectively. In this way the flux of paired CFL strangelets is lower than those without pairing, but only for low baryon number $(A<\sim 13)$, that is, in a region where it is believed strangelets are not stable at all. In the stability region the flux for CFL strangelets is always higher than for normal strangelets resulting in a higher trapped density. In this way, the smaller difference seeing between strangelets with and without pairing for the improved flux when comparing to that for the standard flux is explained by the smaller difference in the incoming flux due to the softer dependence on the atomic number (the standard flux depends on $E^{-2.5}$, which for the same rigidity depends on the particle's atomic number; instead, the improved flux depends on $R^{1.8}$ and the analysis was performed in terms of same rigidity).

Additional considerations are relevant for the fate of a trapped population of strangelets. It is well-known that the solar wind has a strong influence on the ACR flux upon the earth. The most abundant ACR heavy ion, oxygen, shows a strong intensity variation with the solar cycle, having its interstellar flux of $8-27 \mathrm{MeV} /$ nucleon lowered up to two orders of magnitude during periods of solar maximum activity [56]. During solar minimum, the trapped flux at the earth magnetosphere is of the order of $\sim 5 \times 10^{-4}$ particles $\mathrm{cm}^{-2} \mathrm{sr}^{-1} \mathrm{~s}^{-1}(\mathrm{MeV} / \text { nucleon })^{-1}$, corresponding to an enhancement factor of $\sim 15$ [57], this experimental value being somewhat below the theoretical expected one (higher than 25 [56]). The oxygen component corresponds to about $80 \%$ of the trapped ACR, while the $\mathrm{C} / \mathrm{O}, \mathrm{N} / \mathrm{O}$ and $\mathrm{Ne} / \mathrm{O}$ abundance ratio are $<0.005, \sim 0.10-0.15$ and $\sim 0.02-0.03$, respectively.

With the results obtained in this study, the trapped flux of strangelets at $L<2$ would be of order $10^{-14}-10^{-15}$ particles $\mathrm{cm}^{-2} \mathrm{sr}^{-1} \mathrm{~s}^{-1}(\mathrm{MeV} / \mathrm{A})^{-1}$ at rigidity $R=1 G V$ for strangelets of baryon number $A=100$. This represents an enhancement factor for 
trapped flux in the regime of steady-state population comparing to the interstellar flux at the same energy and $A$ of order 10 and $10^{2}$ for strangelets trapped at $L=2$ and $L=1.3$, respectively, the values for CFL strangelets being of about twice the one for normal strangelets ( $q \sim 5.5$ and 11, and $q \sim 162$ and 314 for CFL and normal strangelets at $L=2$ and $L=1.3$, respectively). This results show that the strangelet flux could be as high as a factor 10000 lower than that expected for carbon during periods of maximum solar activity. Although we did not consider the solar modulation in our calculations, it would act significantly over those low energy strangelets [40], the region of interest in this study. In this manner, it could have an important influence, similar to that detected for oxygen, on the trapped density.

The advantage of a search for trapped strangelets in the geomagnetic field performed during the solar maximum activity whether they are an important component of the radiation belt or are to be measured penetrating the atmosphere towards to surface of the earth would be the reduced component of ACR, which could reduce dead time losses in the detectors and possibly render a clearer identification of the primaries.

The proposed and widely accepted model for ACR trapping [58] assumes that the high mass-to-charge ratio of singly-ionized ACRs enables them to penetrate deeply into the magnetosphere. ACRs with trajectories near a low altitude mirror point interact with particles in the upper atmosphere, loosing one or all their remaining electrons. After stripping, the particle gyroradius is reduced by a factor of $1 / Z$, and the ion can become stably trapped. As stated above, the results presented here were obtained assuming fully-ionized strangelets, which have just the "right" features to become trapped. However, some fraction of the strangelets should reach earth's atmosphere with an effective charge slightly below their atomic number and suffer a process of interaction similar to ACR's, which is much less dependent on the pitch angle and other variables. Finally, there is also the possibility of quasi-stable trapping of ions with energies high enough not to obey condition (7), but not too high as to penetrate the magnetosphere without suffering any significant depletion in their incident direction. These two additional mechanisms could result in a further increase in the number of trapped strangelets.

\section{Conclusions}

From the analysis presented here we conclude that nonrelativistic strangelets with $A<\sim 10^{3}$ already ionized by collisions with electrons in the ISM could be stably trapped by the geomagnetic field. Assuming the existence of a strangelet contamination in the ISM, its injection in the solar system and given the geomagnetic geometry and the interaction of the magnetic field with the solar wind, it looks very likely to have this radiation belt surrounding the planet. If strangelets are to be a component of the anomalous cosmic ray belt at $L \sim 2$, we have shown that, even considering the approximations taken during the calculations presented here (which have the main consequence of averaging the trapped population's behavior), those particles would be present with an enhancement factor comparing with the interstellar flux of order $10^{1}$ and if we consider a new particle belt (a strangelet belt) at $L \sim 1.3$, the enhancement factor could be as high as order $10^{2}$ in a stationary population scenario [61]. These exotic baryons could in principle be detectable in the earth magnetosphere depending on the chosen parameters for each of the experiments (effective detection area, altitude and type of orbiting, magnetic field for particle depletion and others). In addition to the already mentioned capture of almost fully ionized strangelets, additional trajectories leading to trapping (but not obeying the adiabatic conditions) may exist, although they must be calculated numerically, and could enhance even further the trapped population, though most probably not affecting substantially the results. Effects that could result in the reduction of the trapped population are the diffusion driven by electric fields fluctuations and phenomena directly related to enhanced solar activity, which though less likely to affect the particles already trapped at low $L$-shells, could have an influence on the particle injection in the outer magnetosphere.

Overall, we believe our estimates to be on the conservative side of the trapped flux, making the search of trapped strangelets a feasible but difficult task. Needless to say, the detection of those trapped particles having low $Z / A$ ratio would be extremely important for determining the properties of cold, dense baryonic matter.

\section{Acknowledgments}

L.P. acknowledges the financial support received from the Fundação de Amparo à Pesquisa do Estado de São Paulo. J.E.H. wishes to acknowledge the CNPq Agency (Brazil) for partial financial support.
[1] E. Witten, Phys. Rev. D 30, 272 (1984).

[2] A. Bodmer, Phys. Rev. D 4, 1601 (1971).

[3] S. A. Chin and A. Kerman, Phys. Rev. Lett. 43, 1292 (1979).
[4] H. Terazawa, Tokyo U. Report. pp. INS-336 (1979).

[5] E. Farhi and L. Jaffe, Phys. Rev. D 30, 2379 (1984).

[6] E. P. Gilson and R. L. Jaffe, Phys. Rev. Lett. 71, 332 (1993). 
[7] J. Madsen, astro-ph/9809032.

[8] M. Buballa, hep-ph/0402234.

[9] G. Lugones and J. Horvath, Phys. Rev. D 66, 074017 (2002).

[10] M. Alford, K. Rajagopal, and F. Wilczek, Nucl. Phys. B 537, 433 (1999).

[11] R. Rapp et al., Ann. Phys. (N. Y.) 280, 35 (2000).

[12] K. Rajagopal and F. Wilczeck, for an overview of the CFL state, hep-ph/0011333.

[13] J. Madsen, J.Phys. G 28, 1737 (2002).

[14] R. Klingenberg, J. Phys. G 27, 475 (2001).

[15] E. Finch, nucl-ex/0605010.

[16] J. Thomas and P. Jacobs, A guide to the high energy ion experiments, UCRL-ID-119181.

[17] A. Rusek et al. (for the E886 collaboration), Phys. Rev. C 54, R15 (1996).

[18] G. Van Buren (for the E864 collaboration), J. Phys. G 25, 411 (1999).

[19] J. Belz et al. (for the BNL E888 collaboration), Phys. Rev. Lett. 76, 3277 (1996).

[20] F. Dittus et al. (for the NA52 collaboration), in International Conference on Strangeness in Hadronic Matter, edited by J. Rafelski (American Institute of Physics, New York (AIP 340), 1995), p. 24.

[21] G. Appelquist et al., Phys. Rev. Lett. 76, 3907 (1996).

[22] G. Ambrosini et al., Nucl. Phys. A610, 306c (1996).

[23] R. Klingenberg, J. Phys. G 25, R273 (1999).

[24] R. Arsenescu et al., New J. Phys. 4, 96 (2002).

[25] A. De Rujula and S. L. Glashow, Nature 312, 734 (1984).

[26] M. Bruegger et al., Nature 337, 434 (1989).

[27] M. C. P. Isaac et al., astro-ph/9806147.

[28] Z.-T. Lu et al., nucl-ex/0402015.

[29] J. D. Bjorken and L. McLerran, Phys. Rev. D 20, 2353 (1979).

[30] M. Rybczynski, Z. Wlodarczyk, and G. Wilk, hepph/0109225.

[31] T. Saito et al., Phys. Rev. Lett. 65, 2094 (1990).

[32] M. Rybczynski, Z. Wlodarczyk, and G. Wilk, hep$\mathrm{ph} / 0410065$.

[33] V. Choutko (for the AMS-01 Collaboration), in Proc. 28th Internat. Cosmic Ray Conf., Tsukuba, Japan (Universal Academic Press, Tokyo, 2003), p. 1765.

[34] J. E. Horvath and L. Paulucci, J. Phys. G 32, B13 (2006).

[35] V. Kalogera et al., Astrophys. J. Lett. 601, L179 (2004).

[36] W. Kluzniak and W. Lee, MNRAS 335, L29 (2002).

[37] F. Limousin, D. Gondek-Rosinska, and E. Gourgoulhon, gr-qc/0411127.

[38] O. G. Benvenuto and J. E. Horvath, Phys. Rev. Lett. 63, 716 (1989).
[39] G. A. Medina-Tanco and J. E. Horvath, Astrophys. J. 464, 364 (1996).

[40] J. Madsen, Phys. Rev. D 71, 014026 (2005).

[41] H. C. Rosu, hep-ph/9410028.

[42] Home page of AMS experiment, URL http://ams.cern.ch

[43] J. Madsen, hep-ph/0111417.

[44] K. Rajagopal and F. Wilczek, Phys. Rev. Lett. 86, 3492 (2001).

[45] J. Madsen and J. M. Larsen, Phys. Rev. Lett. 90, 121102 (2003).

[46] C. Lattes and K. Fujimoto, Phys. Repts. 65, 151 (1980).

[47] M. W. Werner and E. E. Salpeter, MNRAS 145, 249 (1969).

[48] H. S. Massey and E. H. S. Burhop, Electronic and Ionic Impact Phenomena (Oxford University Press, 1952).

[49] H. A. Bethe and E. E. Salpeter, Handbuch der Physik, vol. 35 (Springer-Verlag Berlin, 1957).

[50] N. Bohr, Kgl. Dansk. Vid. Selsk. 18, 8 (1948).

[51] C. Stoermer, The polar aurora (Claredon Press, Oxford, 1955).

[52] J. H. Adams, R. Silberberg, and C. H. Tsao, Tech. Rep. NRL Memorandum Report 4506, Naval Research Laboratory, Washington DC 20375-500, USA (1981), See also, for example, D. F. Smart and M. A. Shea, Adv. Space Res. 14(10), 787 (1994).

[53] A. J. Tylka, in Proc. 23rd Internat. Cosmic Ray Conf., Calgary, Canada (1993), vol. 3, p. 436.

[54] M. Walt, Introduction to geomagnetically trapped radiation (Cambridge Press, 1994).

[55] W. Baumjohann, private communication.

[56] S. Biswas, Space Science Rev. 75, 423 (1996).

[57] V. V. Bobrovskaya et al., Cosmic Research 39, 98 (2001).

[58] J. B. Blake and L. M. Friesen, in Proc. 15th Internat. Cosmic Ray Conf., Plovdiv, Bulgaria (1977), vol. 2, p. 341

[59] C.-G. Falthammar, in Earth's Particles and Fields (Ed.B.M. McCormac, Reinhold Book Co., 1968), pp. 157-169.

[60] Radial diffusion caused by random variations in the potential electric fields have a softer dependence on the McIlwain parameter, being the resultant diffusion coefficient $D_{L L} \propto L^{6}[59]$.

[61] It must be noted, however, that for the ACR belt the theoretical values for the enhancement factor are somewhat higher than the experimental ones, a feature that would probably hold for the strangelet belt as well. 